\title{
Viscoelasticity measured by shear wave elastography in a rat model of NAFLD: comparison with dynamic mechanical analysis
}

\section{Zhaoke Pi}

Shenzhen University

Mengke Wang

Shenzhen University

Haoming Lin

Shenzhen University

Yanrong Guo

Shenzhen University

\section{Siping Chen}

Shenzhen University

Xianfen Diao

Shenzhen University

Hui Xia

Institute of Electrical Engineering Chinese Academy of Sciences

Guoqiang Liu

Institute of Electrical Engineering Chinese Academy of Sciences

Jie Zeng

Third Affiliated Hospital of Sun Yat-Sen University

Xinyu Zhang

Shenzhen University

Xin Chen ( $\nabla$ chenxin@szu.edu.cn )

Shenzhen University

\section{Research}

Keywords: Nonalcoholic fatty liver disease, shear wave elastography, Dynamic mechanical analysis, Viscoelasticity

Posted Date: March 24th, 2020

DOl: https://doi.org/10.21203/rs.3.rs-18971/v1 
License: (c) (i) This work is licensed under a Creative Commons Attribution 4.0 International License. Read Full License 
1 Viscoelasticity measured by shear wave elastography

2 in a rat model of NAFLD: comparison with dynamic

3 mechanical analysis

4

5

6

7

8

9

Zhaoke $\mathrm{Pi}^{1}$, Mengke Wang ${ }^{1}$, Haoming Lin ${ }^{1}$, Yanrong Guo ${ }^{1}$, Siping Chen ${ }^{1}$, Xianfen Diao ${ }^{1}$, Hui Xia ${ }^{2}$, Guoqiang Liu ${ }^{2}$, Jie Zeng ${ }^{3}$, Xinyu Zhang ${ }^{1 *}$, Xin Chen ${ }^{1 *}$

${ }^{1}$ School of Biomedical Engineering, Health Center, Shenzhen University, NationalRegional Key Technology Engineering Laboratory for Medical Ultrasound, Guangdong Key Laboratory for Biomedical Measurements and Ultrasound Imaging, Shenzhen 518000, China

${ }^{2}$ Institute of Electrical Engineering, Chinese Academy of Sciences, Beijing 100000, China

3 Department of Medical Ultrasonics, Third Affiliated Hospital of Sun Yat-Sen University, Guangzhou 510000, China

${ }^{*}$ Correspondence should be addressed to Xinyu Zhang; xyzhang9@ szu.edu.cn and Xin Chen; chenxin@szu.edu.cn 


\section{Abstract}

Background: Nonalcoholic fatty liver disease (NAFLD) is rapidly becoming one of the most common liver diseases. Ultrasound elastography has been used for the diagnosis of NAFLD. However, clinical research on steatosis by elastography technology has mainly focused on steatosis with fibrosis or non-alcoholic steatohepatitis (NASH), while steatosis without fibrosis has been poorly studied. Moreover, the relationship between liver viscoelasticity and steatosis grade is not clear. In this study, we evaluated the degree of liver steatosis in a simple steatosis rat model using shear wave elastography (SWE).

Results: The viscoelasticity values of 69 rats with hepatic steatosis were measured quantitatively by SWE in vivo and validated by a dynamic mechanical analysis (DMA) test. Pathological sections were used to determine the steatosis grading for each rat. The results showed that the elasticity values obtained by the two methods followed the same trend, and the elasticity is significantly correlated with liver steatosis. The Pearson's correlation coefficients indicate that elasticity obtained by SWE is positively linear correlated with DMA $\left(\mathrm{r}=0.628, p=7.85 \times 10^{-9}\right)$

Conclusion: SWE might have the feasibility to be introduced as an auxiliary technique for NAFLD patients in clinical settings. However, the viscosity results measured by the two methods are significantly different because the two methods work in different frequency bands.

Keywords: Nonalcoholic fatty liver disease, shear wave elastography, Dynamic mechanical analysis, Viscoelasticity

\section{Background}

Nonalcoholic fatty liver disease (NAFLD) is a main cause of chronic liver disease (CLD) and is rapidly becoming one of the most common liver diseases, with an approximate prevalence of $20 \%-30 \%$ of the total population in Western countries [1, 2]. It represents a spectrum of diseases ranging from simple steatosis to nonalcoholic steatohepatitis (NASH), which has a close relationship with liver cirrhosis and hepatocellular carcinoma [3-5]. NASH is critical in the development of liver fibrosis and liver failure and has become a new challenge in the field of liver disease research.

Many different techniques have been studied in the diagnostic of NAFLD. Magnetic resonance elastography (MRE) uses a modified phase-contrast method to image the propagation characteristics of the shear wave in the liver. Early researches of MRE suggest that it could accurately differentiate simple steatosis from NASH with or without fibrosis. However, MRI is costly and time consuming for use in routine clinical practice [6]. Recently, several ultrasound-based elastography techniques, including transient elastography (TE) [7-9], acoustic radiation force impulse (ARFI) [10] and shear wave elastography (SWE) [11], have been used for the noninvasive diagnosis of liver disease. Compared with liver biopsy or other imaging methods, ultrasound 
elastography has the advantages of a lower cost and a smaller time commitment. As the procedure is non-invasive, it can supersede biopsy-related postoperative complications and potential diagnosis error due to a small sampling range [12].

The mechanical properties of liver tissue may be helpful in the diagnosis of liver diseases. Many studies had shown that liver stiffness measured by elastography techniques is strongly linked to liver fibrosis [13, 14]. The World Federation for Ultrasound in Medicine and Biology (WFUMB) recently produced guidelines which recommended the use of elastography techniques for distinguishing advanced fibrosis $(\geq$ F2) from early fibrosis ( $\leq$ F1) [15]. However, the mechanism of hepatic steatosis development is different from that of fibrosis. Some researches on steatosis by elastography technology mainly focused on steatosis with fibrosis, while steatosis without fibrosis has been poorly studied. The relationship between steatosis severity and liver stiffness has not been determined [16-18]. Many studies have suggested that liver steatosis with the accumulation of lipid deposits may cause changes of viscoelasticity rather than stiffness. Zhu et al. proposed that the characterization of shear wave dispersion is significantly related to various oil percentages in gelatin phantoms [19] .Barry et al. showed that an increase in liver steatosis clearly increased the viscosity in a mouse liver model, ex vivo [20]. Deffieux et al. [21] found no obvious correlation of liver viscosity and dispersion curve slope with steatosis or disease activity. However, the relationship between liver viscoelasticity and steatosis grade is not clear.

Mechanical tests have been used as another method to assess tissue mechanical properties [22-25]. For example, the dynamic mechanical analysis (DMA) is considered a conventional "gold standard" method to assess the mechanical properties of tissues and can determine the viscoelastic properties of tissues. Many studies have used DMA tests to measure the mechanical properties of liver tissue for comparison of various ultrasound elastography technique results [26, 27].

Our previous study showed the feasibility of assessing the viscoelasticity of liver in a rat model of steatosis by the DMA test [25]. In this study, we used shear wave elastography to evaluate the viscoelasticity of rat liver with steatosis. A DMA test was performed by oscillatory shear test on a liver sample from the same rat model as before, and then parameters, including the elasticity and the viscosity, were subjected to comparative analysis with variation in the grade of liver steatosis.

\section{Results}

\section{Histologic and biochemical characteristics}

liver tissue sections from mice in each group after SWE measurements were stained with Oil Red $\mathrm{O}$ and examined under the light microscope. Fat droplets in liver tissue were increased significantly as the stages of steatosis increased. Fig. 1 shows representative histologic findings according to the severity of NAFLD. 


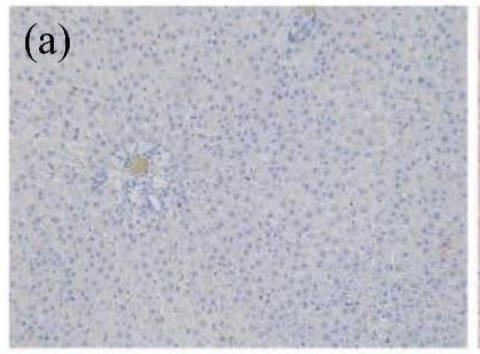

(b)
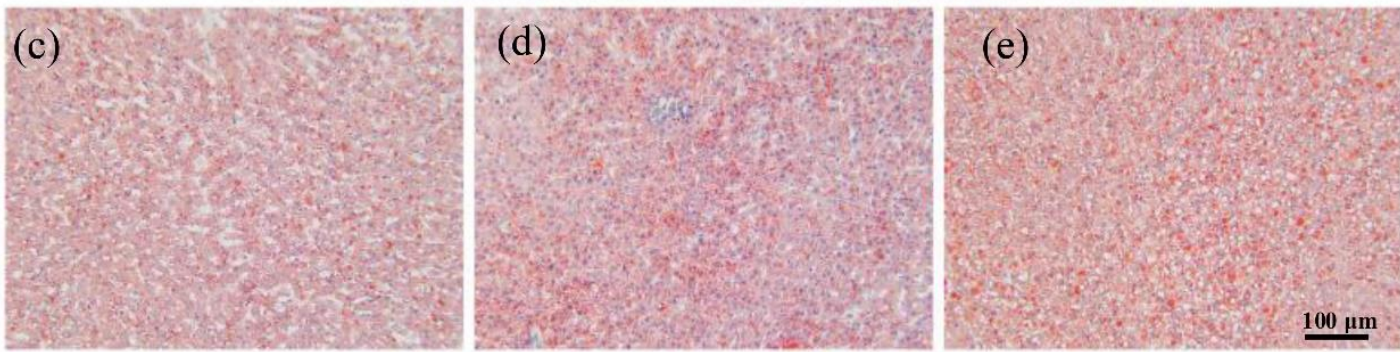

Fig. 1 Typical specimens of five rat livers at different liver steatosis stages. (ae) S0-S4.

\section{Use of SWE and DMA for assessing the grade of steatosis}

The elasticity and viscosity values of 69 rats for S0-S4 obtained by the two methods are shown in Fig. 2. For each stage, the rats in this stage were sorted according to their mean elasticity values obtained by DMA method. The mean elasticity and viscosity values for each stage are listed in Table 1 . The mean values of elasticity obtained by SWE (from $0.86 \pm 0.27 \mathrm{kPa}$ to $1.05 \pm 0.29 \mathrm{kPa}$ ) were slightly lower than those by DMA (from $1.12 \pm 0.21 \mathrm{kPa}$ to $1.39 \pm 0.31 \mathrm{kPa}$ ). For viscosity, the values of DMA (from 5.40 $\pm 0.78 \mathrm{~Pa}^{*} \mathrm{~s}$ to $6.27 \pm 0.96 \mathrm{~Pa}^{*} \mathrm{~s}$ ) were significant greater than those of SWE (from 0.75 $\pm 0.10 \mathrm{~Pa}^{*} \mathrm{~s}$ to $\left.0.92 \pm 0.18 \mathrm{~Pa}^{*} \mathrm{~s}\right)$.

Table 1 The mean viscoelasticity parameters of all rat livers measured by SWE and DMA methods (n represents the number of rats. In SWE, 10 points in each rat liver were measured in vivo, and 3 liver samples were extracted in random from each rat in DMA, values are the mean \pm standard deviation.)

\begin{tabular}{llllll}
\hline $\begin{array}{l}\text { Degree of liver } \\
\text { steatosis }\end{array}$ & $n$ & SWE & \multicolumn{3}{l}{ DMA } \\
\hline S0 & & Elasticity $(\mathrm{kPa})$ & Viscosity $\left(\mathrm{Pa}^{*} \mathrm{~s}\right)$ & Elasticity $(\mathrm{kPa})$ & Viscosity $\left(\mathrm{Pa}^{*} \mathrm{~s}\right)$ \\
S1 & 17 & $0.86 \pm 0.27$ & $0.77 \pm 0.15$ & $1.12 \pm 0.21$ & $5.40 \pm 0.78$ \\
S2 & 14 & $0.93 \pm 0.23$ & $0.76 \pm 0.20$ & $1.25 \pm 0.26$ & $5.77 \pm 0.71$ \\
S3 & 19 & $1.05 \pm 0.29$ & $0.79 \pm 0.14$ & $1.39 \pm 0.31$ & $6.27 \pm 0.96$ \\
S4 & 12 & $1.01 \pm 0.20$ & $0.75 \pm 0.10$ & $1.29 \pm 0.18$ & $5.97 \pm 0.68$ \\
\hline
\end{tabular}




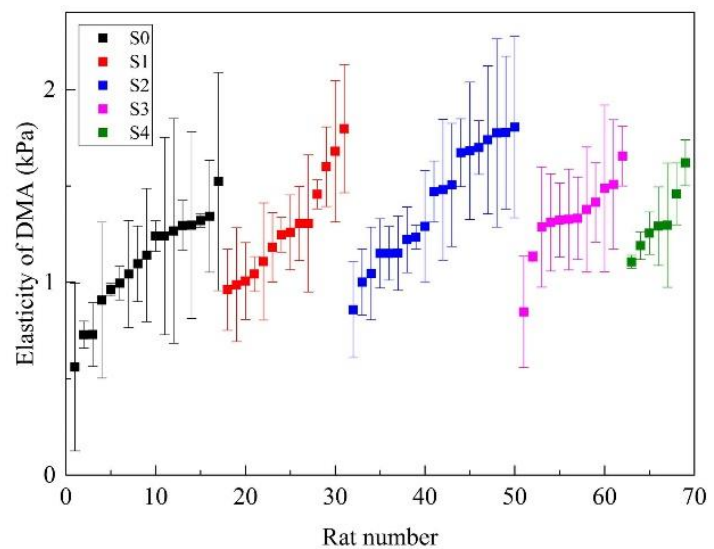

(a)

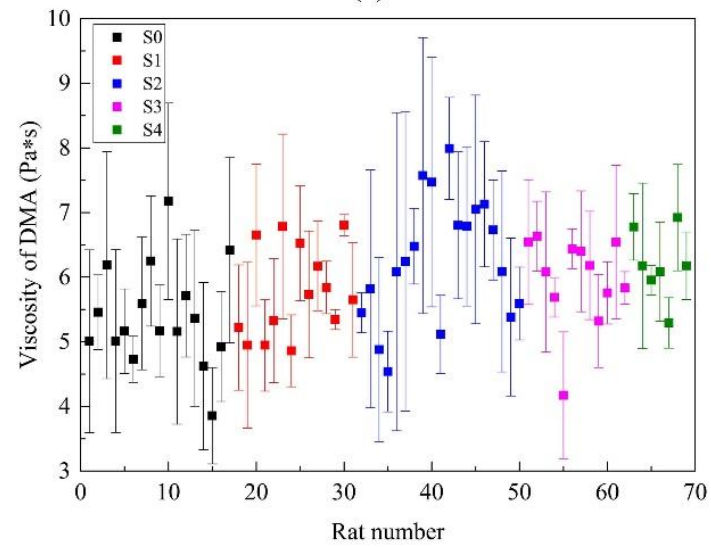

(c)

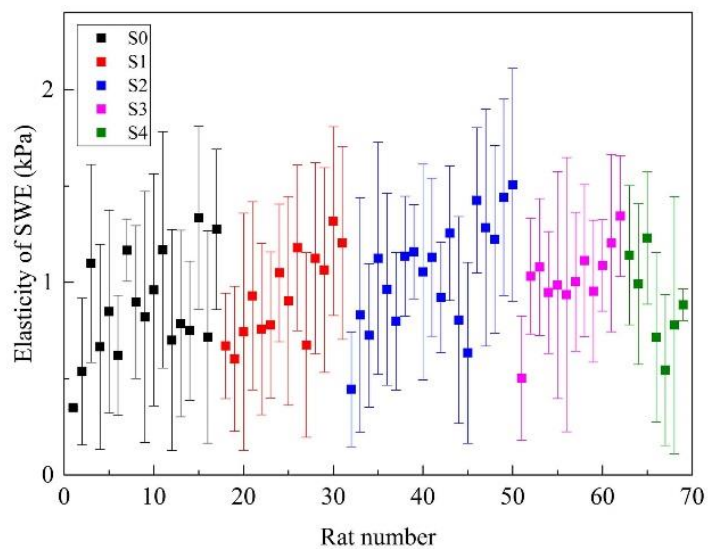

(b)

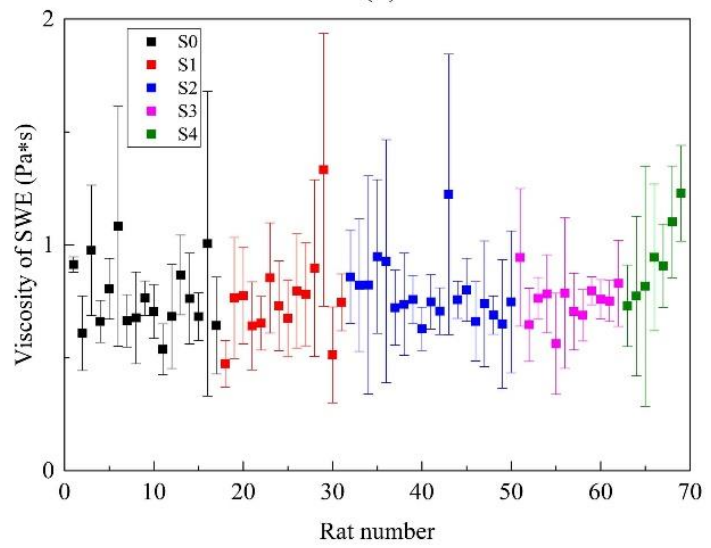

(d)

Fig. 2 The mean and standard deviation of the elasticity and the viscosity in 69 rats for both methods. For each stage, the rats in this stage were sorted according to their mean elasticity values obtained by DMA method.

The box plots in Fig. 3 illustrate the variations of elasticity and viscosity of the two methods for each stage. The results of the ANOVA test with Tukey-Kramer multiple comparison tests found out the group pairs which had significant differences in elasticity or viscosity.

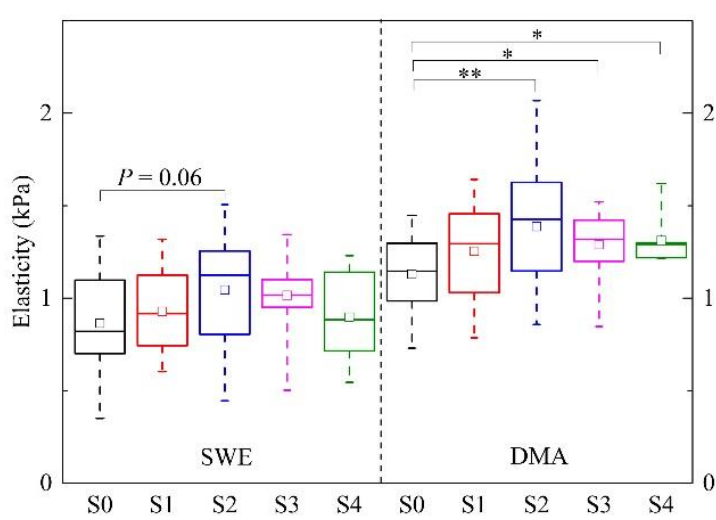

(a)

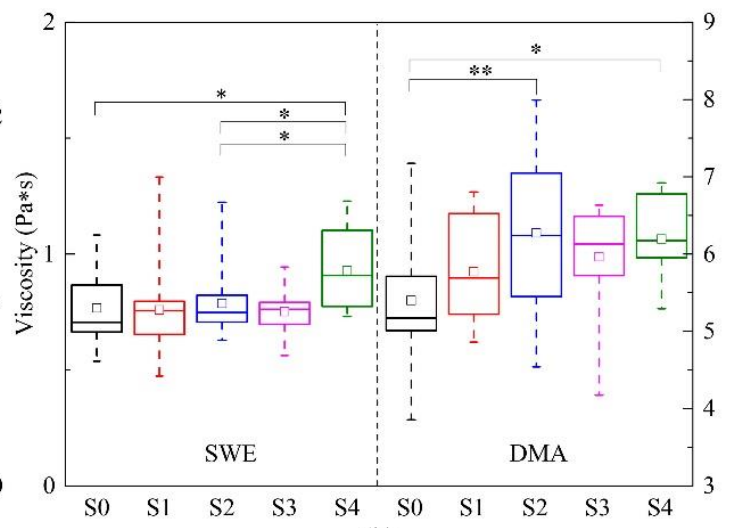

(b)

Fig. 3 Boxplot of (a) the elasticity and (b) the viscosity obtained by the two methods at five steatosis stages. The upper and lower boundaries of the boxes represent the 25th and 75th percentiles, lines within the boxes represent 
medians, squares within the boxes represent means, and error bars represent ranges. Asterisks indicate the pairs having statistically significant differences in the Tukey-Kramer test after the ANOVA test. ${ }^{*} p<0.05,{ }^{* *} p<0.01$.

\section{Correlation of SWE and DMA on the elasticity and the viscosity}

Correlations between the SWE and DMA for the elasticity and viscosity are illustrated in Fig. 4. The Pearson's correlation coefficients indicate that elasticity obtained by SWE is positively linear correlated with DMA $\left(\mathrm{r}=0.628, p=7.85 \times 10^{-9}\right)$, while there is almost no correlation between viscosity obtained by SWE and DMA $(\mathrm{r}=-0.01, p=-0.91)$.

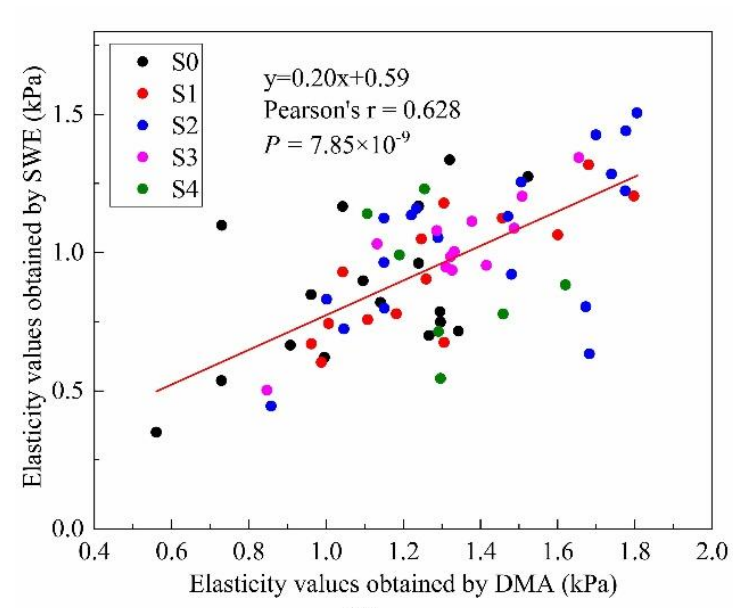

(a)

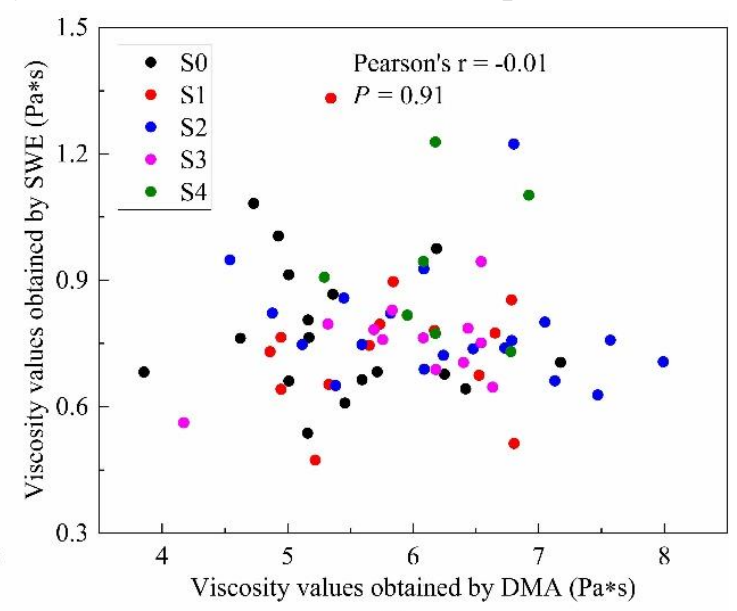

(b)

Fig. 4 Correlative analysis between the SWE and DMA about (a) the elasticity and (b) the viscosity at five steatosis stages.

\section{Discussion}

NAFLD is known to be a complex disorder and includes simple steatosis, NASH and fibrosis. In clinical situations, steastosis and fibrosis are usually interleaved together, and it is difficult to distinguish their separate effects on the mechanical propertiese of the liver tissue. Many clinical researchers of elastography technology have mainly focused on steatosis with fibrosis or NASH. Grimal et al. [28] investigated the relationship between liver tissue stiffness and histological inflammation score, hepatic fibrosis stage, ballooning score, steatosis analyzed by SWE in rats with NASH, and found that median liver stiffness values measured using SWE showed a stepwise increase with increasing steatosis grade $(p=0.03$ ), the results were consistent with those of [29] that demonstrated that liver elasticity was effective in detecting NAFLD. However, Nightingale et al. [30] performed shear wave dispersion with a linear dispersion model to perform shear wave dispersion analysis in traditionally "difficultto-image" subjects in 135 NAFLD patients. Nevertheless, viscoelastic parameters were not related to the steatosis stage. The presence of entirely different results in the relationship between liver viscoelasticity and steatosis stage may have many causal factors, such as different frequency ranges corresponding to different viscoelastic parameters, experimental conditions, machines and operators. Moreover, the role of 
SWE measurement in vivo is more complicated because of the complex structure of the organism and variable factors. It also becomes difficult to evaluate when a patient has more than just a single disease in the clinical setting. Parker et al. [31] summarized numerous studies about ultrasound shear wave dispersion in healthy and steatosis livers and concluded that animal models and human populations need further investigation.

In this study, we used a rat model of liver steatosis, which avoided the confounder of liver fibrosis, thus we can focus on studying the effect of steatosis on viscoelasticity. Disease development in the soft tissue is always accompanied by a variety of mechanical properties of the tissues. The viscoelastic properties of tissue are promising for utilizing the inherent mechanical properties of tissue for grading disease. To this end, we performed liver viscoelasticity measurements using SWE in rat models with various degrees of steatosis severity. The DMA tests were used as a standard of reference, and the values of elasticity and viscosity obtained by SWE were compared to the degree of steatosis as evaluated by histological assessment. In this study, we used the Voigt model to obtain the viscoelasticity parameters in a rat model of NAFLD. We found that the values of elasticity obtained by SWE increased from stage S0 to S2 (Fig. 3(a)), which was similar to the trend of DMA. Interestingly, the values decrease slightly from stage $\mathrm{S} 2$ to $\mathrm{S} 4$, which is also similar to the DMA. Although the trend between the values of elasticity obtained by SWE is consistent with DMA, the $p$ values of elasticity obtained by SWE were slightly greater than 0.05 and not statistically different among the five steatosis stages. The elasticity of DMA was statistically different between stages $\mathrm{S} 0$ and S2-S4. The values of elasticity obtained by SWE were relatively lower than those obtained by DMA. However, compared with the viscosity of DMA, the results of SWE were significantly lower, and there was no difference from $\mathrm{S} 0$ to $\mathrm{S} 3$. The mean value of S4 was higher than other stages (Fig. 3(b)). The trends of the five stages of viscosity obtained by SWE were not consistent with those obtained by DMA [19]. Although some phantom studies have shown that viscosity can provide relevant and independent information about the inherent state, until now, few researchers could determine the viscoelasticity parameters in vivo for the diagnosis of fatty liver disease.

Despite the results obtained by SWE based on shear wave dispersion, accurate staging of the grade of liver steatosis did not prove statistically significant. However, it is interesting to evaluate the values of viscoelasticity relationships between SWE and DMA. In Fig. 4, the mean values of SWE were highly correlated with those obtained by DMA; the correlation coefficient was 0.628 , and $p=7.85 \times 10^{-9}$, indicating significant linear correlation. However, the mean values of viscosity measurements obtained by SWE were relatively independent of those obtained by DMA with a correlation coefficient of -0.01 . This result suggests that the elasticity may provide useful information about the liver steatosis status obtained by SWE. There is little relationship between SWE and DMA in terms of viscosity, suggesting that it has a weak correlation with liver steatosis, which is consistent with previously reported results [21].

The viscosity results measured by the two methods are significantly different, because 
the measurements depend heavily on the frequency bands. It is focused to investigate why the elasticity values measured by SWE have the same trend with the DMA but without a statistical difference among the five steatosis stages. There may be several reasons for this result. First, DMA has much lower frequencies than SWE, which may lead to different vibrations in microstructures of liver tissue. Also, the frequency ranges of the shear wave measured using SWE may not reveal the actual viscoelastic properties of the tissue[32]. In DMA tests, the frequency ranged from 1 to $41 \mathrm{~Hz}$, while the shear wave frequency of SWE ranged from 160 to $380 \mathrm{~Hz}$. Specifically, the excised liver has no blood circulation in the DMA tests. Blood flow can affect the viscoelasticity measurement of the liver by SWE [33]. In addition, the limited size of the species utilized and ultrasound imaging quality considerations can also affect the results.

\section{Conclusion}

In this study, we compared the measurement of viscoelastic parameters, including elasticity and viscosity, by DMA tests and SWE methods in a rat model of NAFLD at five steatosis stages. The results showed that the elasticity values from the two methods followed the same trend, suggesting that elasticity has a significant correlation with liver steatosis. To some extent, elasticity values measured by SWE can reflect liver steatosis processes. SWE might have the feasibility to be introduced as an auxiliary technique for NAFLD patients in clinical settings.

\section{Materials and Methods}

\section{Animal model}

A total of 69 male Sprague-Dawley (SD) rats (weighing $200 \pm 20$ g, Guangdong Medical Laboratory Animal Center, Foshan, China) were used in this study. They were maintained under Specific Pathogen Free (SPF) conditions (relatively constant temperature: $20-26^{\circ} \mathrm{C}$ humidity: $30-50 \%, 10 \mathrm{~h}: 14 \mathrm{~h}$ light/dark rhythm alternately) with a high-calorie diet. To induce hepatic steatosis in different stages (S1-S4), a highcalorie diet for various numbers of days were supplied to SD rats at a dose of $1 \mathrm{~mL} / 100$ $\mathrm{g}$ rat weight once a day for sixty days. The control group (S0) was fed with a regular diet. Rat livers were harvested for DMA tests and histologic assessment after the rat had been used for in vivo ultrasound measurements. The histopathologic examination ultimately determined the hepatic steatosis stage. The number of animals in each group was stated in Table 1. Animal care and experiments were approved by the Animal Care and Use Committee of School of Medicine in Shenzhen University and Guangdong Medical Laboratory Animal Center. 


\section{In vivo SWE measurement}

In this experiment, the rat was placed in the supine position on the experimental table after anesthesia at a relatively constant temperature of $24^{\circ} \mathrm{C}-26^{\circ} \mathrm{C}$. The rat abdomen was shaved and coated with ultrasonic gel, and then an ultrasound B-mode image was used for localization of the liver and a region of interest (ROI) (size: $5 \mathrm{~mm} \times 15 \mathrm{~mm}$ ) in the median lobe of the liver, devoid of large vessels, was chosen for measurement. After the ROI of the liver was selected, the operator kept the probe stable and switched to the SWE mode to finish the measurement. To obtain a relatively accurate result, for each rat, measurements were repeated ten times in ten locations, and the calculated elasticity and viscosity values were the mean of ten valid measurements.

The SWE measurement was performed on the Verasonic vantage 256 systems (Verasonics Inc., Kirkland, WA, USA) with a linear array transducer L11-4v (Philips Healthcare, Andover, MA). The measurement procedure is similar to that used in the previous study [34]. First, a B mode image of the liver was obtained to provide imaging guidance for selecting the measurement locations, then a region of interest (ROI), $5 \mathrm{~mm}$ axially $\times 15 \mathrm{~mm}$ laterally, was selected for measurement. A total of 20 array elements were used in one excitation, which consisted of three successive push beams to create a Mach cone [35]. These three push beams were focused at $-5 \mathrm{~mm}, 0 \mathrm{~mm}$, and $5 \mathrm{~mm}$ deep relative to the center of the ROI with a time interval of 50 microseconds. The push beam at each depth was generated by an 80 microsecond tone burst at a center frequency of 4.4 MHz. Second, the system was switched immediately to the plane wave imaging mode after the push wave transmission. All elements were set to fire 50 detect pulses with a center frequency of $6.25 \mathrm{MHz}$ and a pulse repetition frequency of $10 \mathrm{kHz}$. Next, the data post-processing steps include: (1) In-phase quadrature (IQ) data within the ROI was processed with a 2D correlation algorithm to obtain particle velocity [36]; (2) a $3 \times 3$ median filter was used to the liver tissue to remove spike noise, cubic spline interpolation with a factor of five was conducted to reconstruct the displacement profiles to increase the temporal resolution; (3) low-pass filtering with a cut off frequency of $1000 \mathrm{~Hz}$ was used to reduce jitter after the reverberation frames were removed, then a Fourier transform was used to estimate the phase at each frequency; (4) then a directional filter was applied to reduce the artifacts from reflected shear waves at boundaries, a linear regression algorithm was used to calculate the shear wave phase velocities at each frequency $(160-380 \mathrm{~Hz})$ by estimating the average phase difference [37]; (5) finally the values of viscoelasticity were estimated by MATLAB (Mathworks, R2014a) with a nonlinear fitting method using Equation 1 based on a least-squares criterion.

\section{Ex vivo DMA experiment}

Each rat liver was harvested immediately for ex vivo DMA test after the in vivo ultrasound measurement. The livers were soaked with saline solution to prevent desiccation of liver specimens and kept in the refrigerator at a temperature of $4^{\circ} \mathrm{C}$ to preserve freshness. 


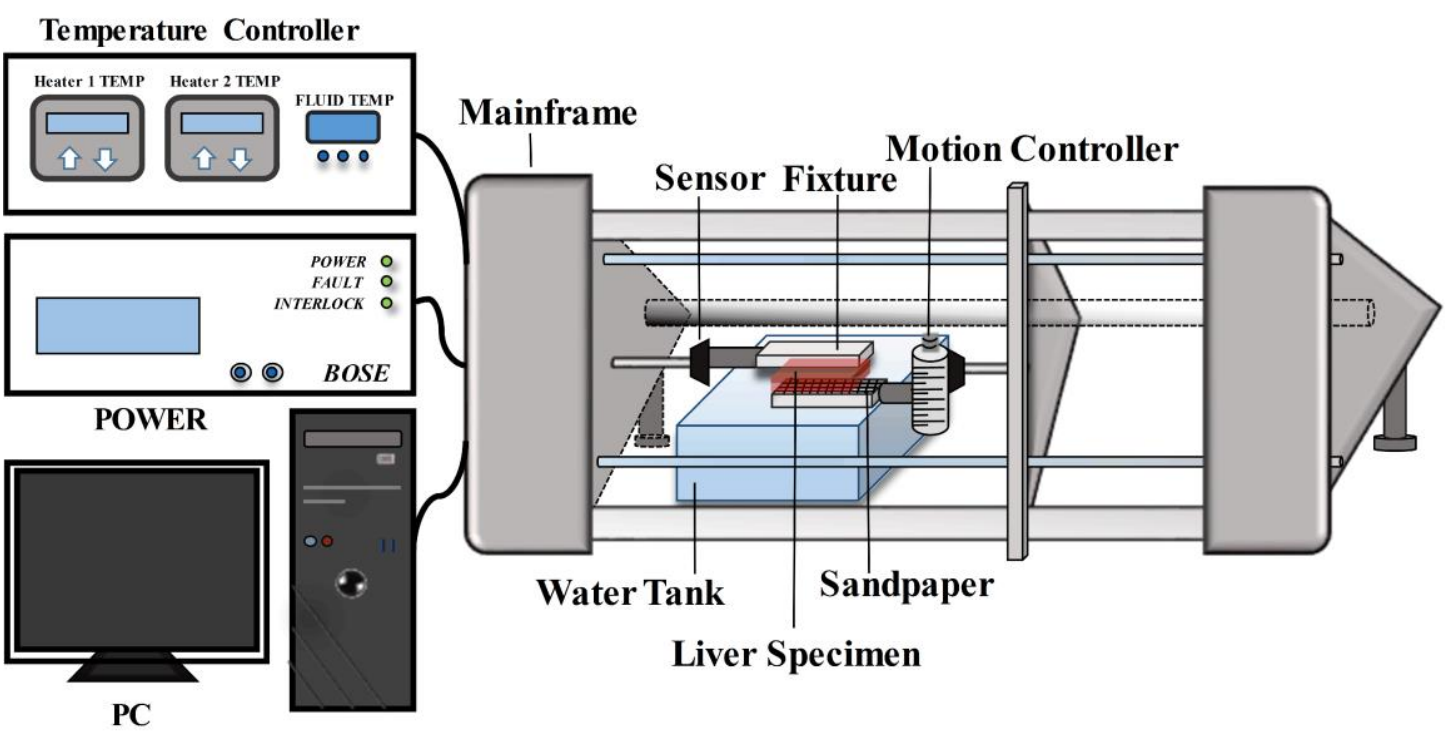

Fig. 5 The dynamic mechanical analysis (DMA) experimental platform.

Fig. 1 shows the DMA experimental instrument (the ElectroForce3200 Series, Boss Corp., Eden Prairie, Minnesota, USA) operating at a parallel plate shear mode. Two to three liver specimens were taken from each rat, and then each specimen was cut by a scalpel into the same shape (size: $15 \mathrm{~mm} \times 5 \mathrm{~mm} \times 4 \mathrm{~mm}$, thickness: $4 \pm 1 \mathrm{~mm}$ ) to match the parallel plates. During the experiment, the liver specimen was fixed to the fixture in the water tank which was filled with a saline solution at a body temperature of $38.1 \pm$ $0.2^{\circ} \mathrm{C}$. Sandpaper was attached between the parallel plates to prevent the liver specimen from slipping. The motion controller was rotated slowly to ensure sufficient contact between the parallel plates. After the sample was placed on the parallel plates, the sample slowly received a precompression of 2-3 g to ensure sufficient contact between the plate and the sample and avoid slippage. A sinusoidal torque was engendered by the machine to make the liver specimen generate stress and strain. Then, the dynamic mechanical properties, including storage modulus $G^{\prime}$ and loss modulus $G^{\prime \prime}$ were directly obtained from the system during the test. The phase velocities at all frequency points in the range of $1-41 \mathrm{~Hz}$ with an interval of $5 \mathrm{~Hz}$ were substituted in Equation 3 and Equation 3, and then shear elasticity $\mu$ and shear viscosity $\eta$ of every sample were estimated by non-linear curve-fitting.

\section{Methods}

In this study, it is assumed that the liver consists of viscoelastic and isotropic homogenous material. The relationship between the shear wave velocity and frequency was derived from the Voigt model. The equation is as shown below [38]: 


$$
c_{s}(\omega)=\sqrt{\frac{2\left(\mu^{2}+\omega^{2} \eta^{2}\right)}{\rho\left(\mu+\sqrt{\mu^{2}+\omega^{2} \eta^{2}}\right)}}
$$

where $c_{s}$ is the shear velocity, $\omega$ the angular frequency, $\rho$ the mass density, $\mu$ and $\eta$ are the shear elasticity and shear viscosity.

The principle of DMA is performed as previously described [25]. Briefly, for homogeneous biological tissue, the sinusoidal shear strain $\varepsilon(t)=\varepsilon_{0} e^{j \omega t}$ induces a corresponding sinusoidal stress $\sigma(t)=\sigma_{0} e^{j(\omega t+\sigma)}$. The complex shear modulus $G^{*}$ is defined as the ratio of stress to the strain:

$$
G^{*}(\omega)=\frac{\sigma(t)}{\varepsilon(t)}=\frac{\sigma_{0} e^{j(\omega t+\sigma)}}{\varepsilon_{0} e^{j \omega t}}=\frac{\sigma_{0}}{\varepsilon_{0}}(\cos \delta+j \sin \delta)=G^{\prime}(\omega)+j G^{\prime \prime}(\omega)
$$

Where $\omega$ is angular frequency, $\delta$ is a phase difference, $\sigma_{0}$ and $\varepsilon_{0}$ are the shear stress amplitude and strain amplitude, respectively. The real part of the Equation 2, $G^{\prime}(\omega)$ is the storage modulus relating to the elasticity parameter, $\mu$, while the imaginary part $G^{\prime \prime}(\omega)$ is the loss modulus relating to the viscosity $\eta$. To quantitatively analyze the viscoelastic properties of the tissues, the complex modulus is expressed by $G^{*}=\mu+j \omega \eta$, which is derived from the Voigt model [39]. Therefore, the storage moduli and loss moduli of the liver are expressed as follows:

$$
G^{\prime}=\mu
$$

\section{Histological assessment}

The rest of each rat liver tissue was fixed in the $10 \%$ neutral formalin liquid, and conventional methods of dehydration, paraffin-embedded sections and Oil Red O staining were conducted by histopathology technicians in the Guangdong Medical Laboratory Animal Center. Two slices, with a thickness of $7 \mu \mathrm{m}$, from each rat were used for the histologic examination. These pathological slices were observed under an optical microscope by pathologists who had no knowledge of the in vivo ultrasound measurement. Stages of steatosis were determined by quantifying percentages of hepatocellular macrovesicular steatosis: 0-4\% (S0); 5-25\% (S1); 26-50\% (S2); 51-75\% (S3); $>76 \%$ (S4). Fig. 1 shows typical liver specimens for the five steatosis stages. 


\section{Statistical analysis}

All 69 rats were divided into five groups separated by histological grade (S0-S4). For each steatosis grade, the viscoelasticity parameter values of all the rats at that grade were grouped. The mean parameter value of each rat was then used to estimate the population mean of each steatosis group. If the value exceeded more than 1.5 times the interquartile range in its group, it was eliminated as an outlier and the corresponding measurement was excluded [25]. The relationship between the SWE and DMA on the elasticity and the viscosity was analyzed using the Pearson correlation coefficient and linear regression. All data were represented as means \pm standard deviation (SD) and analyzed by using the two-tailed student t-test, analysis of variance. The one-way analysis of variance (ANOVA) with the Tukey-Kramer multiple comparison tests was used to compare the liver viscoelasticity parameters among the steatosis stages. Differences were considered significant when ${ }^{*} p<0.05, * * p<0.01$, and $* * * p<0.001$, respectively.

\section{Abbreviations}

NAFLD: nonalcoholic fatty liver disease; NASH: non-alcoholic steatohepatitis; SWE: shear wave elastography; DMA: dynamic mechanical analysis; MRE: magnetic resonance elastography; TE: transient elastography; ARFI: acoustic radiation force impulse; SD: Sprague-Dawley; SPF: Specific Pathogen Free; ROI: region of interest; IQ: in-phase quadrature; ANOVA: analysis of variance.

\section{Authors' contributions}

Zhaoke Pi and Haoming Li designed the method and drafted the original manuscript; Mengke Wang, Yanrong Guo, Xianfen Diao, and Jie Zeng participated in the experiments and analyzed the data; Siping Chen, Hui Xia, Guoqiang Liu, Xinyu Zhang and Xin Chen provided supports for the research, designed the method, and revised the manuscript. All authors read and approved the final manuscript.

\section{Availability of data and materials}

The data used to support the findings of this study are available from the corresponding author upon reasonable request.

\section{Ethics approval and consent to participate}

Animal care and experiments were approved by the Animal Care and Use Committee of School of Medicine in Shenzhen University, China (Approval No.: 20160110). 


\section{Consent for publication}

2 All authors give their consent for publication.

\section{Competing interests}

$4 \quad$ The authors declare that they have no conflict of interest.

\section{$5 \quad$ Acknowledgments\&Funding}

This work was supported by the National Natural Science Foundation of China (Grant No. 81601503, 61427806, 81601510, 81570552, 61101025,), National Natural Science Foundation of Guangdong Province (Grant No. 2016A030310047), Postgraduate Innovation Development Fund of Shenzhen University (Grant No. 315-0000470204).

\section{References}

1. Bellentani S, Scaglioni F, Marino M, Bedogni G. Epidemiology of Non-Alcoholic Fatty Liver Disease. Digest Dis. 2010; 28(1):155-61.

2. de Alwis NMW, Day CP. Non-alcoholic fatty liver disease: The mist gradually clears. J Hepatol. 2008; 48:S104-12.

3. Dowman JK, Tomlinson JW, Newsome PN. Pathogenesis of non-alcoholic fatty liver disease. QJM. 2010; 103(2):71-83.

4. Powell EE, Jonsson JR, Clouston AD. Dangerous liaisons: The metabolic syndrome and nonalcoholic fatty liver disease. Ann Intern Med. 2005; 143(10):753-4.

5. Vernon G, Baranova A, Younossi ZM. Systematic review: the epidemiology and natural history of non-alcoholic fatty liver disease and non-alcoholic steatohepatitis in adults. Aliment Pharm Ther. 2011; 34(3):274-85.

6. Talwalkar JA, Meng Y, Glaser KJ, Sanderson SO, Ehman RL. Early detection of nonalcoholic steatohepatitis in patients with nonalcoholic fatty liver disease by using MR elastography. Radiology. 2011; 259(3):749-56.

7. Castera L, Forns $X$, Alberti A. Non-invasive evaluation of liver fibrosis using transient elastography. J Hepatol. 2008; 48(5):835-47.

8. Sandrin L, Fourquet B, Hasquenoph JM, Yon S, Fournier C, Mal F, et al. Transient elastography: A new noninvasive method for assessment of hepatic fibrosis. Ultrasound Med Biol. 2003; 29(12):1705-13.

9. Wang MH, Palmeri ML, Guy CD, Yang L, Hedlund LW, Diehl AM, et al. In Vivo Quantification of Liver Stiffness in a Rat Model of Hepatic Fibrosis with Acoustic Radiation Force. Ultrasound Med Biol. 2009; 35(10):1709-21.

10. Nightingale $K$, Soo MS, Nightingale $R$, Trahey G. Acoustic radiation force impulse imaging: In vivo demonstration of clinical feasibility. Ultrasound Med Biol. 2002; 28(2):227-35. 
11. Sarvazyan AP, Rudenko OV, Swanson SD, Fowlkes JB, Emelianov SY. Shear wave elasticity imaging: A new ultrasonic technology of medical diagnostics. Ultrasound Med Biol. 1998; 24(9):1419-35.

12. Ratziu V, Charlotte F, Heurtier A, Gombert S, Giral P, Bruckert E, et al. Sampling variability of liver biopsy in nonalcoholic fatty liver disease. Gastroenterology. 2005; 128(7):1898-1906.

13. Friedrich-Rust M, Wunder K, Kriener S, Sotoudeh F, Richter S, Bojunga J, et al. Liver Fibrosis in Viral Hepatitis: Noninvasive Assessment with Acoustic Radiation Force Impulse Imaging versus Transient Elastography. Radiology. 2009; 252(2):595-604.

14. Braticevici CF, Sporea I, Panaitescu E, Tribus L. Value of Acoustic Radiation Force Impulse Imaging Elastography for Non-Invasive Evaluation of Patients with Nonalcoholic Fatty Liver Disease. Ultrasound Med Biol. 2013; 39(11):1942-50.

15. Ferraioli G, Filice C, Castera L, Choi BI, Sporea I, Wilson SR, et al. Wfumb Guidelines and Recommendations for Clinical Use of Ultrasound Elastography: Part 3: Liver. UI Ultrasound Med Biol. 2015; 41(5):1161-79.

16. Ebinuma $\mathrm{H}$, Saito $\mathrm{H}$, Komuta M, Ojiro K, Wakabayashi K, Usui S, et al. Evaluation of liver fibrosis by transient elastography using acoustic radiation force impulse: comparison with Fibroscan (R). J Gastroenterol. 2011; 46(10):1238-48.

17. Motosugi $U$, Ichikawa $T$, Niitsuma $Y$, Araki T. Acoustic radiation force impulse elastography of the liver: can fat deposition in the liver affect the measurement of liver stiffness? Jpn J Radiol. 2011; 29(9):639-43.

18. Poynard T, Munteanu M, Luckina E, Perazzo H, Ngo Y, Royer L, et al. Liver fibrosis evaluation using real-time shear wave elastography: Applicability and diagnostic performance using methods without a gold standard. J Hepatol. 2013; 58(5):928-35.

19. Zhu Y, Dong CF, Yin Y, Chen X, Guo YR, Zheng Y, et al. The Role of Viscosity Estimation for Oil-inGelatin Phantom in Shear Wave Based Ultrasound Elastography. Ultrasound Med Biol. 2015; 41(2):601-9.

20. Barry CT, Mills B, Hah Z, Mooney RA, Ryan CK, Rubens DJ, et al. Shear Wave Dispersion Measures Liver Steatosis. Ultrasound Med Biol. 2012; 38(2):175-82.

21. Deffieux $T$, Gennisson JL, Bousquet $L$, Corouge $M$, Cosconea $S$, Amroun $D$, et al. Investigating liver stiffness and viscosity for fibrosis, steatosis and activity staging using shear wave elastography. J Hepatol. 2015; 62(2):317-24.

22. Pailler-Mattei $\mathrm{C}$, Bec $\mathrm{S}$, Zahouani $\mathrm{H}$. In vivo measurements of the elastic mechanical properties of human skin by indentation tests. Med Eng Phys. 2008; 30(5):599-606.

23. Grimal $Q$, Haupert S, Mitton D, Vastel L, Laugier P. Assessment of cortical bone elasticity and strength: Mechanical testing and ultrasound provide complementary data. Med Eng Phys. 2009; 31(9):1140-7.

24. Zhai L, Palmeri ML, Bouchard RR, Nightingale RW, Nightingale KR. An integrated indenter-ARFI imaging system for tissue stiffness quantification. Ultrasonic Imaging. 2008; 30(2):95-111.

25. Zhang XY, Gao XH, Zhang PP, Guo YR, Lin HM, Diao XF, et al. Dynamic mechanical analysis to assess viscoelasticity of liver tissue in a rat model of nonalcoholic fatty liver disease. Med Eng Phys. 2017; 44:79-86.

26. Chatelin S, Oudry J, Perichon N, Sandrin L, Allemann P, Soler L, et al. In vivo liver tissue mechanical properties by transient elastography: Comparison with dynamic mechanical analysis. Biorheology. 2011; 48(2):75-88.

27. Zhu $Y$, Zhang $X Y$, Zheng $Y$, Chen $X$, Shen $Y Y$, Lin HM, et al. Quantitative analysis of liver fibrosis in rats 
with shearvvave dispersion ultrasound vibrometry: Comparison with dynamic mechanical analysis. Med Eng Phys. 2014; 36(11):1401-7.

28. Ogawa S, Moriyasu F, Yoshida K, Oshiro H, Kojima M, Sano T, et al. Relationship between liver tissue stiffness and histopathological findings analyzed by shear wave elastography and compression testing in rats with non-alcoholic steatohepatitis. J Med Ultrason. 2016; 43(3):355-60.

29. Kang BK, Lee SS, Cheong H, Hong SM, Jang K, Lee MG. Shearwave Elastography for Assessment of Steatohepatitis and Hepatic Fibrosis in Rat Models of Non-Alcoholic Fatty Liver Disease. Ultrasound Med Biol. 2015; 41(12):3205-15.

30. Nightingale KR, Rouze NC, Rosenzweig SJ, Wang MH, Abdelmalek MF, Guy CD, et al. Derivation and Analysis of Viscoelastic Properties in Human Liver: Impact of Frequency on Fibrosis and Steatosis Staging. IEEE T Ultrason Ferr. 2015; 62(1):165-75.

31. Parker KJ, Partin A, Rubens DJ. What Do We Know About Shear Wave Dispersion in Normal and Steatotic Livers? Ultrasound Med Biol. 2015; 41(5):1481-7.

32. Wang $Y$, Jiang J. Influence of Tissue Microstructure on Shear Wave Speed Measurements in Plane Shear Wave Elastography: A Computational Study in Lossless Fibrotic Liver Media. Ultrason Imaging. 2018; 40(1):49-63.

33. Asano K, Ogata A, Tanaka K, Ide Y, Sankoda A, Kawakita C, et al. Acoustic Radiation Force Impulse Elastography of the Kidneys Is Shear Wave Velocity Affected by Tissue Fibrosis or Renal Blood Flow? J Ultras Med. 2014; 33(5):793-801.

34. Deng YF, Rouze NC, Palmeri ML, Nightingale KR. Ultrasonic Shear Wave Elasticity Imaging Sequencing and Data Processing Using a Verasonics Research Scanner. IEEE T Ultrason Ferr. 2017; 64(1):164-76.

35. Bercoff J, Tanter M, Fink M. Supersonic shear imaging: A new technique for soft tissue elasticity mapping. IEEE T Ultrason Ferr. 2004; 51(4):396-409.

36. Pinton GF, Dahl JJ, Trahey GE. Rapid tracking of small displacements with ultrasound. IEEE T Ultrason Ferr. 2006; 53(6):1103-17.

37. Deffieux T, Gennisson JL, Bercoff J, Tanter M. On the Effects of Reflected Waves in Transient Shear Wave Elastography. IEEE T Ultrason Ferr. 2011; 58(10):2032-35.

38. Oestreicher HL. Field and Impedance of an Oscillating Sphere in a Viscoelastic Medium with an Application to Biophysics. J Acoust Soc Am. 1951; 23(6):707-14.

39. Macosko CW. Rheology: Principles, Measurements, and Applications. New York: Wiley-VCH, 1994. 


\section{Figures}
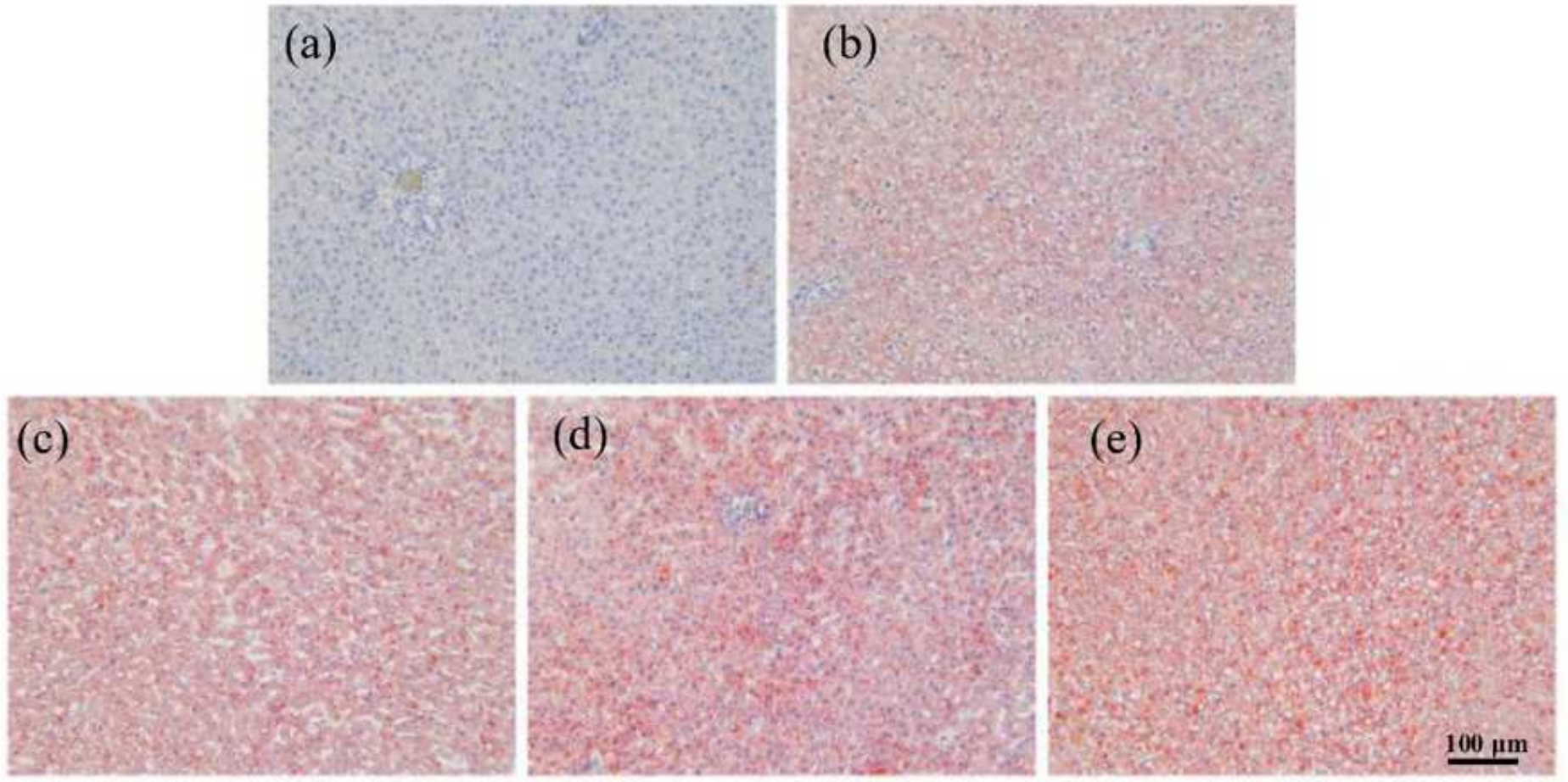

Figure 1

Typical specimens of five rat livers at different liver steatosis stages. (a-e) S0-S4. 


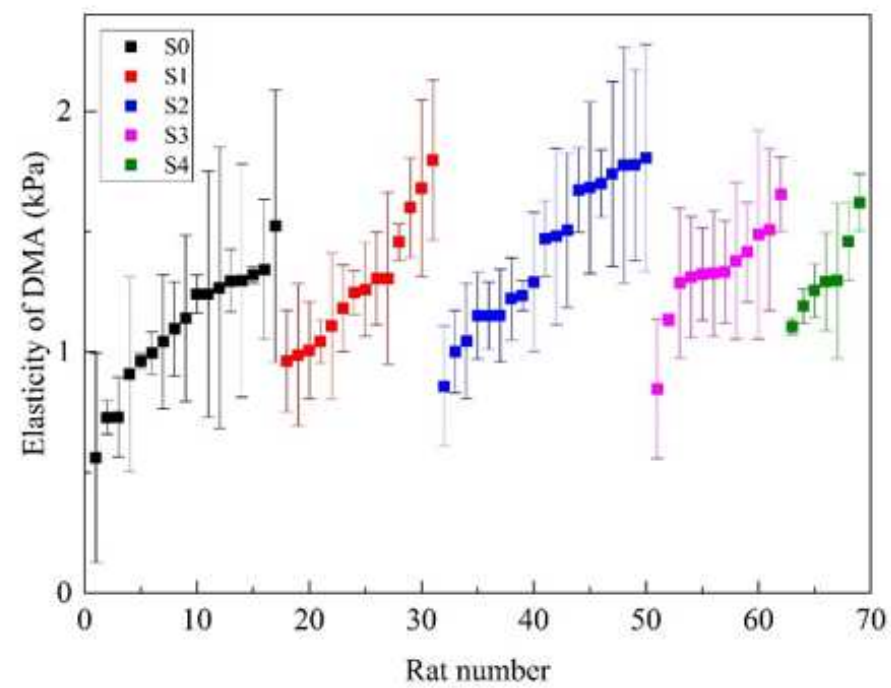

(a)

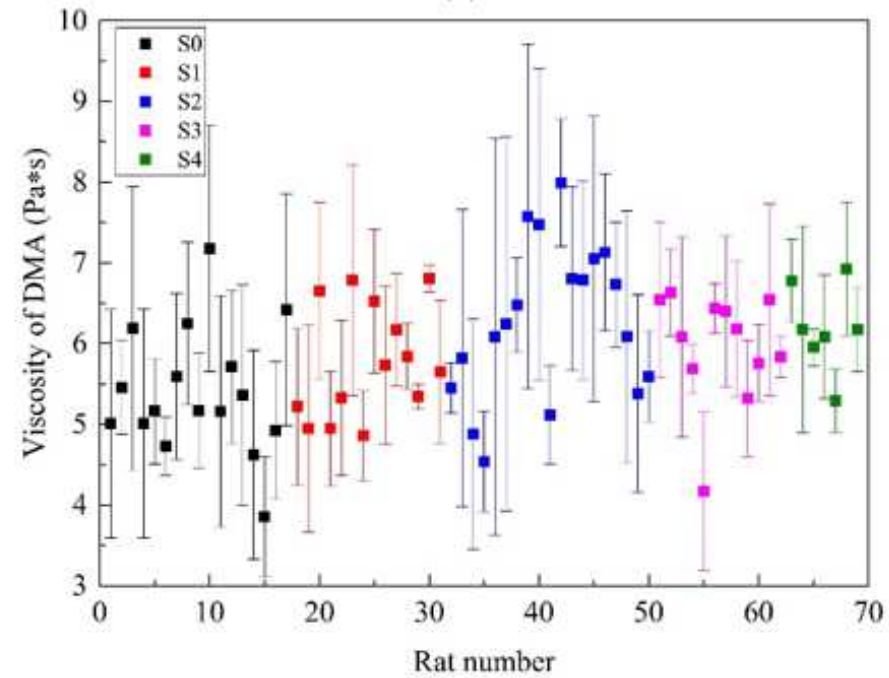

(c)

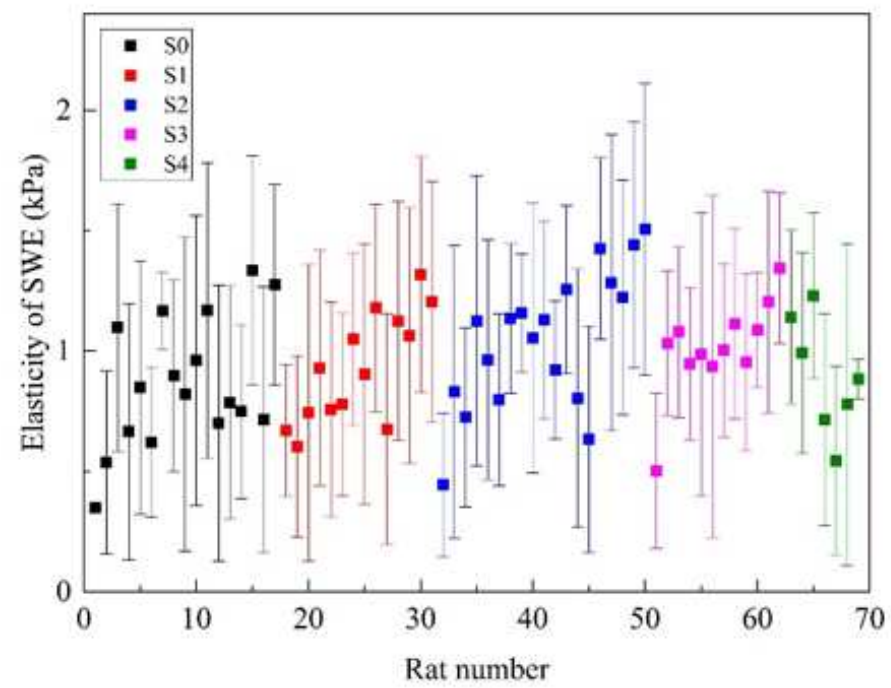

(b)

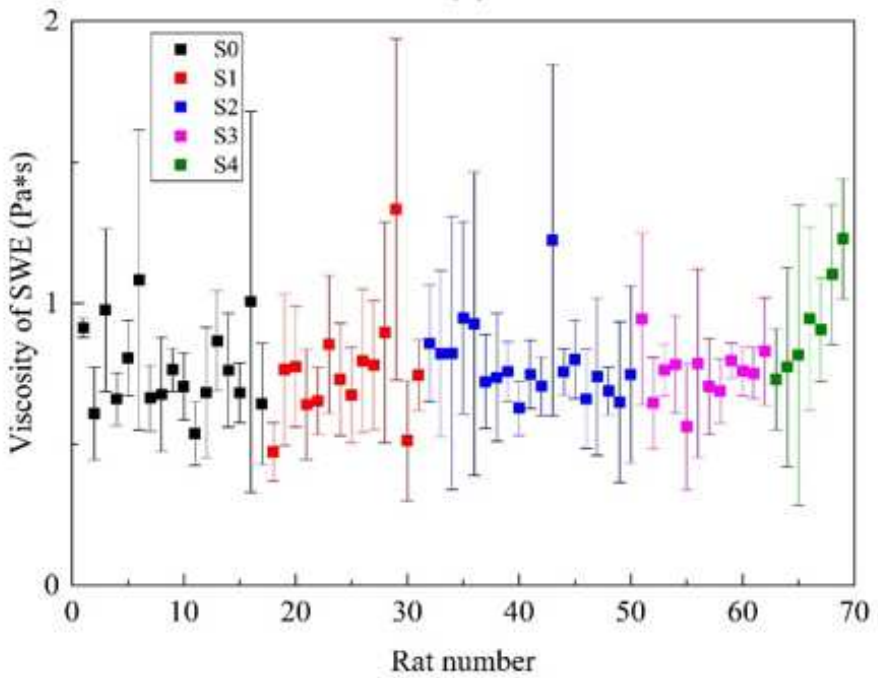

(d)

Figure 2

The mean and standard deviation of the elasticity and the viscosity in 69 rats for both methods. For each stage, the rats in this stage were sorted according to their mean elasticity values obtained by DMA method. 


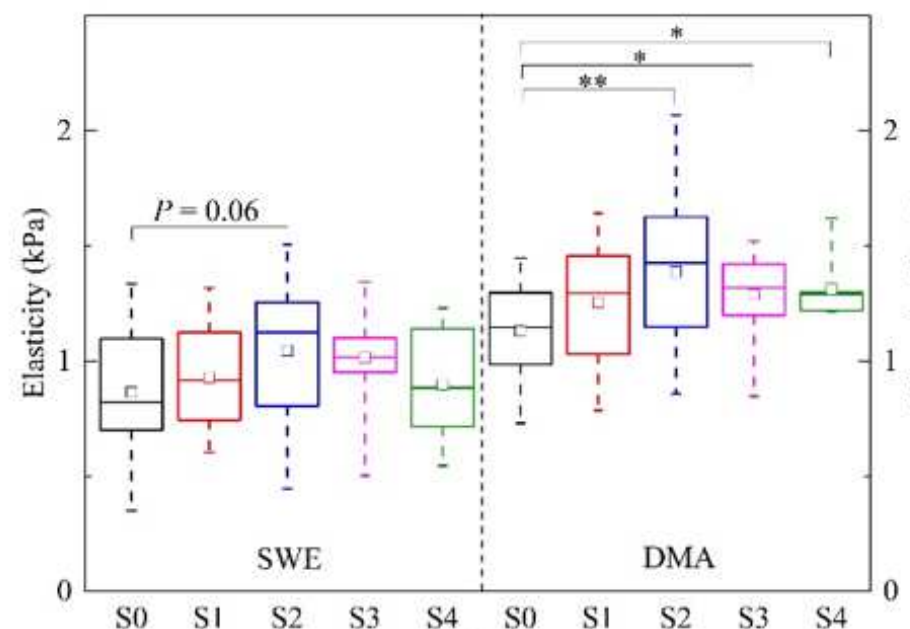

(a)

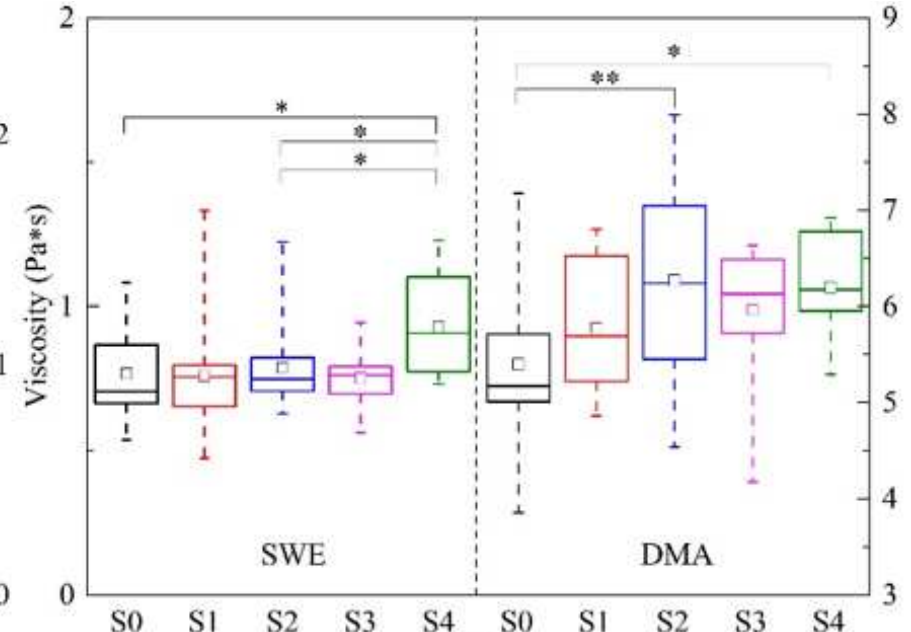

(b)

\section{Figure 3}

Boxplot of (a) the elasticity and (b) the viscosity obtained by the two methods at five steatosis stages. The upper and lower boundaries of the boxes represent the 25th and 75th percentiles, lines within the boxes represent medians, squares within the boxes represent means, and error bars represent ranges. Asterisks indicate the pairs having statistically significant differences in the Tukey-Kramer test after the ANOVA test. ${ }^{*} p<0.05,{ }^{*} \mathrm{p}<0.01$.

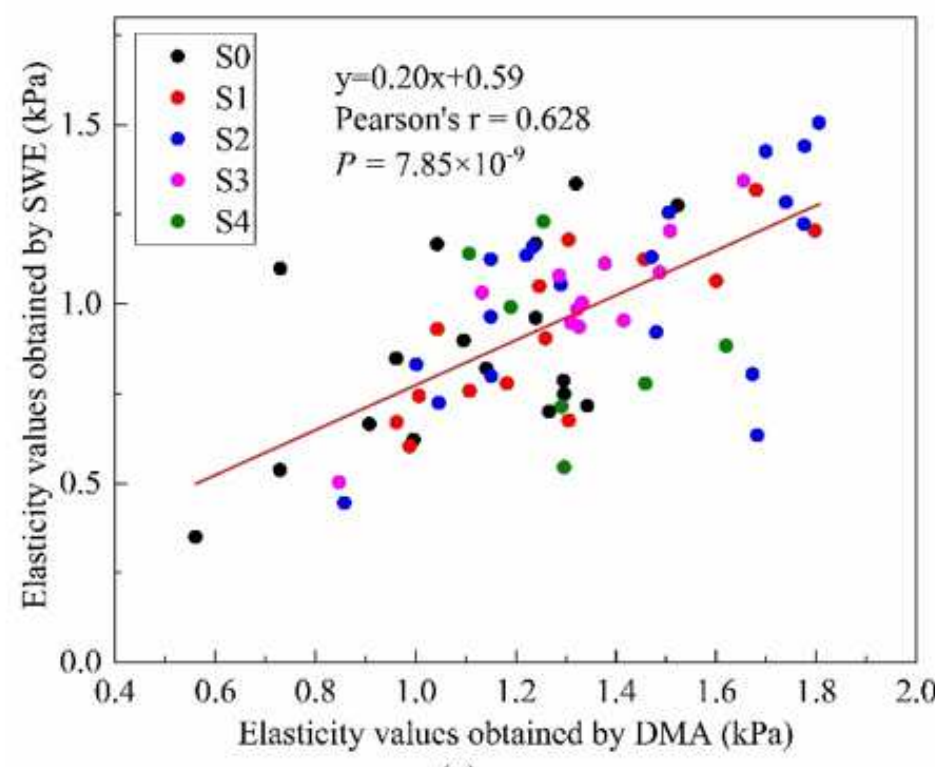

(a)

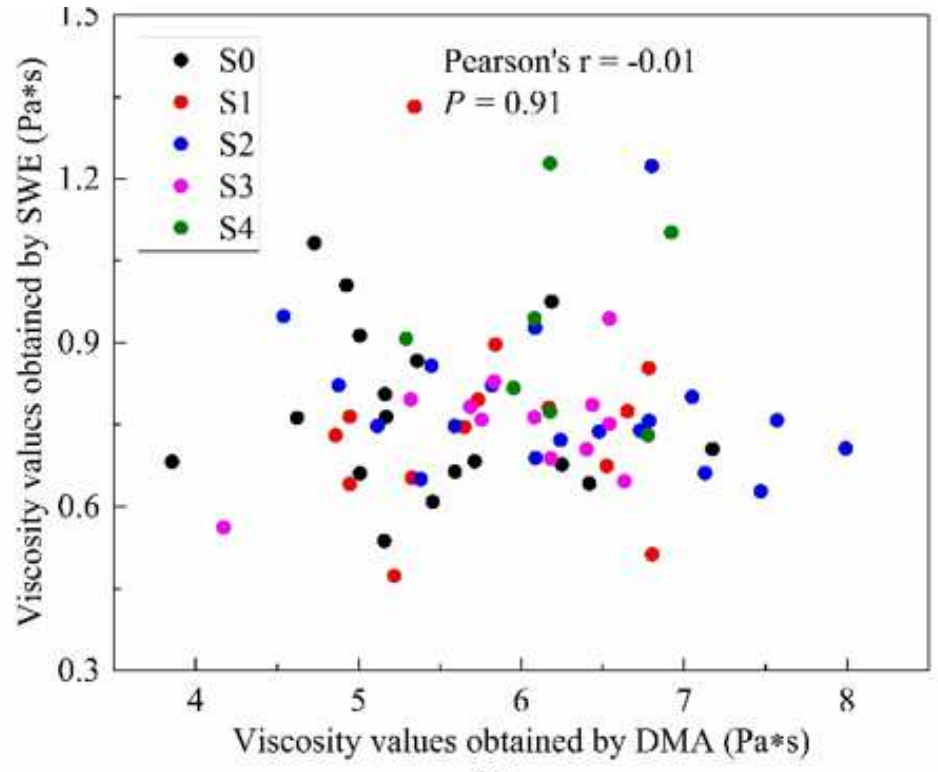

(b)

\section{Figure 4}

Correlative analysis between the SWE and DMA about (a) the elasticity and (b) the viscosity at five steatosis stages. 
Temperature Controller

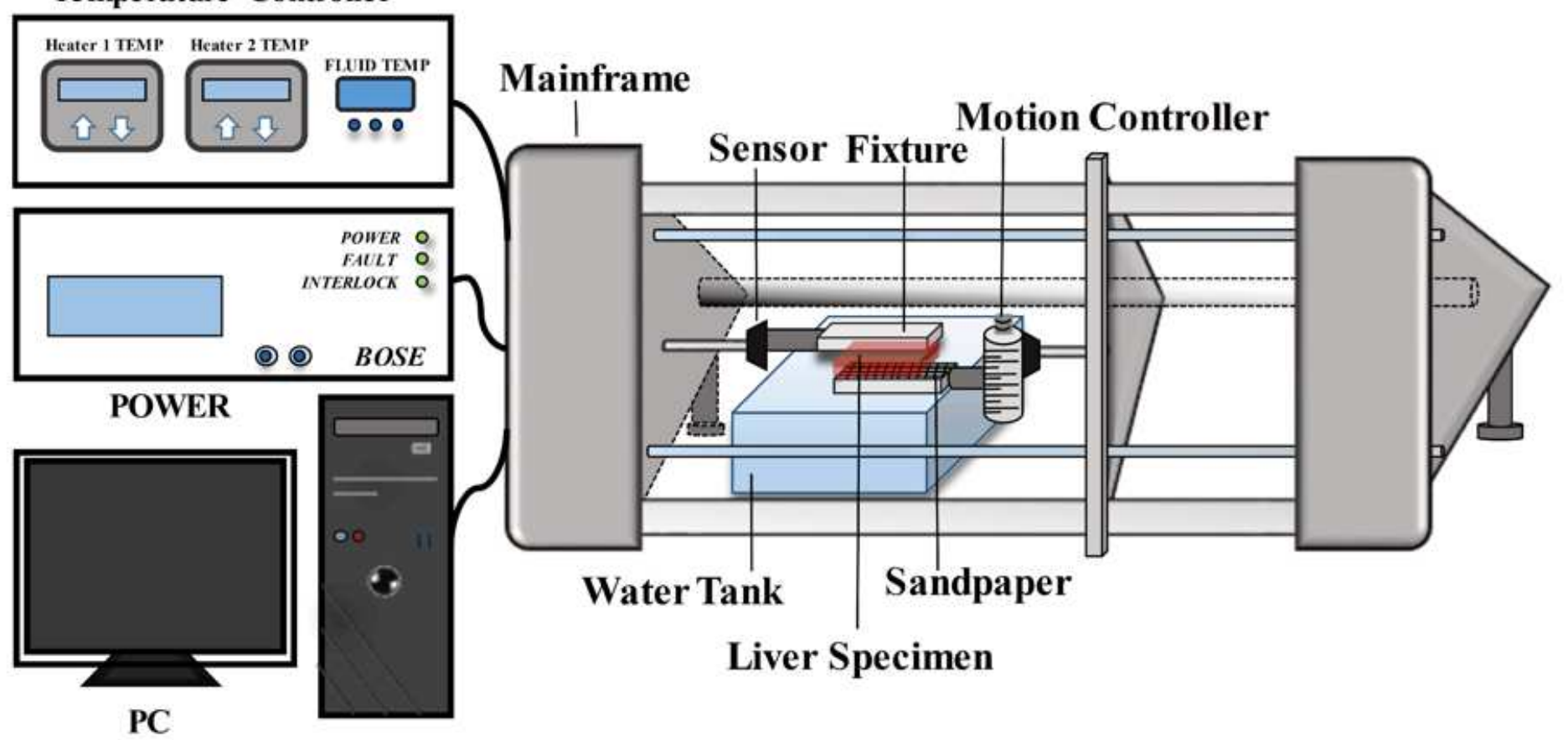

Figure 5

The dynamic mechanical analysis (DMA) experimental platform. 